\title{
Calendrier des événements
}

23 février 2020, Vienne

Initiating Practice Related Research (Lancement de la recherche liée à la pratique) Intervenants : Matthias Desmet (Belgique) et autres

Organisateur : EAP

Info : www.europsyche.org

Contact : peter.schulthess@europsyche.org

29 février 2020, Zurich

Formation postgrade / continue : Approches et méthodes en psychothérapie : Procédures humanistes et intégratives Intervenants : Peter Schulthess

Organisateur : ASP

Info : www.psychotherapie.ch

1 mars 2020, Zurich

Formation postgrade / continue : Approches et méthodes en psychothérapie : Approches systémiques Intervenants : Martin Rufer Organisateur : ASP

Info : www.psychotherapie.ch

20-23 février 2020, Vienne, Autriche

Réunion de l'EAP

Organisateur : EAP (European

Association for Psychotherapy)

Info : www.europsyche.org

28 mars 2020, Zurich

Conférence : L'académisation

de la formation postgrade en psychothérapie - Comment cela

affecte-t-il les prestataires privés?

Organisateur : ASP

Info : www.psychotherapie.ch

17 avril 2020 ; 17h15-19h15, Zurich 43e Assemblée des membres ASP Organisateur : ASP

Info : www.psychotherapie.ch

18 avril 2020, Zurich

Colloque ASP : Science

Organisateur : ASP

Info : www.psychotherapie.ch
24-26 avril 2020, Varsovie, Pologne 4ème Conférence Internationale Organisateur : Scientific Society for Psychodynamic Psychotherapy Info : http://psychoterapiaszkolenia.pl /invitation-to-the-conference

Samedi 25 avril 2020, Zurich

Formation postgrade / continue :

Particularités de la psychothérapie auprès des personnes âgées

Intervenants : Bettina Ugolini

et Claudia King

Organisateur : ASP

Info : www.psychotherapie.ch

Dimanche 26 avril 2020, Zurich

Formation postgrade / continue : Approches et méthodes en psychothérapie :

Thérapie comportementale et méthodes intégratives

Intervenants : Franz Caspar

Organisateur : ASP

Info : www.psychotherapie.ch

17-20 juin 2020, Amherst, Massachusetts, USA 51e Rencontre internationale annuelle de la SPR

Organisateur : SPR

(Society for Psychotherapy Research)

Info : https://www.psychotherapyresearch .org/events/event_list.asp

Samedi 27 juin 2020, Zurich

Colloque ASP : Science

Organisateur : ASP

Info : www.psychotherapie.ch

26-29 juin 2020, Moscou, Russie

9e Congrès mondial de psychothérapie

Organisateur : WCP

(World Council for Psychotherapy)

Info : www.planetofpsychotherapy.com

Samedi 5 septembre 2020, Zurich

Colloque ASP : Science

Organisateur : ASP

Info : www.psychotherapie.ch 